В.Д. ДМИТРИЕНКО, д-р техн. наук, проф., НТУ"ХПИ", Харьков, В.И. НОСКОВ, д-р техн. наук, проф., НТУ"ХПИ", Харьков, B.C. БЛИНДЮК, канд. техн. наук, проф., УкрГАЖТ, Харьков, М.В. ЛИПЧАНСКИЙ, канд. техн. наук, доц., НТУ"ХПИ", Харьков, A.O. НЕСТEРEНКО, асп., НТУ"ХПИ", Харьков

\title{
ОПРЕДЕЛЕНИЕ ОПТИМАЛЬНЫХ ЗАКОНОВ УПРАВЛЕНИЯ ПРОЦЕССАМИ ДВИЖЕНИЯ ЭЛЕКТРОПОЕЗДА
}

Решена задача линеаризации обратной связью по состоянию нелинейной математической модели электропоезда с тяговыми двигателями постоянного тока. На линейной математической модели электропоезда в форме Бруновского решены задачи оптимального управления при разгоне электропоезда. Ил.: 2. Библиогр.: 8 назв.

Ключевые слова: линеаризация обратной связью по состоянию, математическая модель электропоезда, задачи оптимального управления.

Постановка проблемы и анализ литературы. В настоящее время на железных дорогах Украины эксплуатируются электропоезда пригородного сообщения с двигателями постоянного тока, изготовленные свыше 20-25 лет назад. Процесс разгона таких электропоездов осуществляется с помощью системы на основе дополнительных резисторов, на которых выделяется существенное количество энергии. Несмотря на то, что имеются более совершенные системы управления тяговым приводом, установка таких систем на эксплуатируемый подвижной состав не целесообразна из-за высокой стоимости их модернизации. В то же время, по крайней мере, основная часть электропоездов будет эксплуатироваться ещё не менее 8 - 10 лет. В связи с этим возникает проблема совершенствования существующих законов управления электроприводом используемых электропоездов. Вопросами синтеза законов управления дополнительными резисторами занимались многие авторы [1], однако проблема до конца не решена. Это связано с тем, что не только полная модель электропоезда с двигателями постоянного тока слишком сложна для поиска оптимальных законов управления, но и максимально упрощенная модель, полученная на основе замены тягового электропривода одним эквивалентным двигателем постоянного тока с последовательным возбуждением (рис. 1) остается сложной для поиска оптимальных законов управления. 


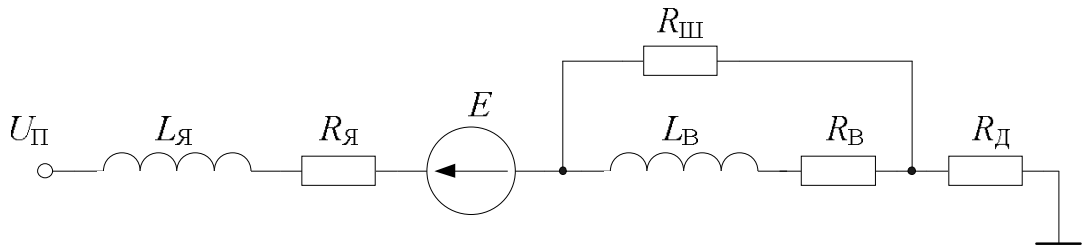

Рис. 1. Эквивалентная схема тягового электропривода электропоезда

На схеме приняты следующие обозначения: $U_{\Pi}-$ напряжение питания эквивалентного двигателя; $L_{Я}, R_{я}, L_{\mathrm{B}}, R_{\mathrm{B}}-$ соответственно индуктивности и активные сопротивления якорной цепи и обмотки возбуждения двигателя; $E$ - электродвижущая сила якоря; $R_{Ш}, R_{\text {Д }}-$ активные сопротивления шунта и дополнительного резистора.

Используя эквивалентную схему тягового электропривода, математическую модель объекта управления можно записать в виде [1]:

$$
\begin{gathered}
\frac{d i_{\text {Я }}}{d t}=\frac{1}{L_{\text {Я }}}\left(U_{\Pi}-\left(R_{\text {Я }}+R_{\amalg}\right) i_{\text {Я }}-R_{\amalg} i_{\mathrm{B}}-C_{\mathrm{E}} \gamma W n\left(1-e^{\beta i_{Я}}\right)-i_{Я} R_{\text {Д }}\right) ; \\
\frac{d i_{\mathrm{B}}}{d t}=\frac{1}{L_{\mathrm{B}}}\left(R_{\amalg} i_{\text {Я }}-\left(R_{\amalg}+R_{\mathrm{B}}\right) i_{\mathrm{B}}\right) ; \\
\frac{d n}{d t}=\frac{\mu}{2 \pi m R_{\mathrm{K}}^{2}}\left(k_{1} C_{\mathrm{M}} i_{Я} \gamma W\left(1-e^{\beta i_{Я}}\right)-\right. \\
\left.-j m g R_{\mathrm{K}} k_{2}\left(a_{0}+a_{1} \frac{2 \pi n R_{\mathrm{K}}}{\mu}+a_{2} \frac{\pi^{2} n^{2} R_{\mathrm{K}}^{2}}{\mu^{2}}\right)\right),
\end{gathered}
$$

где $i_{\text {Я }}, i_{\mathrm{B}}-$ соответственно ток якорной обмотки и ток обмотки возбуждения; $L_{я}, L_{\mathrm{B}}-$ соответственно индуктивности якорной обмотки и обмотки возбуждения; $U_{\Pi}-$ напряжение питания эквивалентного двигателя; $R_{я}, R_{Ш}, R_{\text {Д, }} R_{\mathrm{B}}-$ соответственно активные сопротивления якорной цепи, шунта, дополнительного резистора и обмотки возбуждения; $C_{\mathrm{E}}, \gamma, \beta, C_{\mathrm{M}}, k_{2}, a_{0}, a_{1}, a_{2}$ - постоянные коэффициенты; $W$ - число витков обмотки возбуждения; $n$ - обороты электродвигателя; $\gamma\left(1-e^{\beta i_{я}}\right)$ - аналитическое описание кривой намагничивания; $\mu$ передаточное отношение редуктора электродвигателя; $m$ - масса электропоезда; $R_{\mathrm{K}}$ - радиус колеса колесной пары; $k_{1}$ - число двигателей электропоезда; $j$ - коэффициент, учитывающий уклон железнодорожного пути, в общем случае зависит от участка пути; $g$ - ускорение свободного падения. 
Поскольку объект управления описывается системой нелинейных дифференциальных уравнений третьего порядка, то аналитический синтез регулятора затруднен, хотя возможен, например, с помощью метода аналитического конструирования регуляторов по критерию обобщенной работы $[2,3]$. Однако этот метод предполагает использование трудоемких итерационных процедур расчета матриц коэффициентов. Трудоемких вычислений требуют и другие методы $[4,5]$. В связи с этим актуален поиск методов, решающих задачу оптимального управления тяговым электроприводом электропоезда с приемлемыми затратами машинного времени.

Целью статьи является разработка метода решения задач оптимального управления электропоездом на основе геометрической теории управления.

Геометрическая теория управления предполагает вначале переход с помощью средств дифференциальной геометрии в новое пространство, где математическая модель объекта управления остается эквивалентной исходной модели, но становится линейной. Затем синтез регулятора или законов управления для объекта, который описывается системой линейных обыкновенных дифференциальных уравнений, а потом обратный переход в исходное пространство, где объект описывается системой нелинейных дифференциальных уравнений $[6,7]$.

Запишем систему уравнений (1) привода электропоезда в режиме разгона в более удобной форме:

$$
\begin{aligned}
& \frac{d x_{1}}{d t}=a_{10}+a_{11} x_{1}+a_{12} x_{2}+a_{13} x_{3}+a_{131} x_{3} e^{\beta x_{1}}+a_{11 u} x_{1} u ; \\
& \frac{d x_{2}}{d t}=a_{21} x_{1}+a_{22} x_{2} ; \\
& \frac{d x_{3}}{d t}=a_{30}+a_{31} x_{1}+a_{311} x_{1} e^{\beta x_{1}}+a_{33} x_{3}+a_{333} x_{3}^{2},
\end{aligned}
$$

где $x_{1}=i_{я} ; x_{2}=i_{\mathrm{B}} ; x_{3}=n ; a_{10}, a_{11}, a_{12}, \ldots, a_{33}, a_{333}-$ постоянные коэффициенты; $u=R_{\text {д }}$ - управление.

Необходимым и достаточным условием линеаризации обратной связью по состоянию $[6,7]$ в некоторой окрестности $\Omega$ начала координат математической модели нелинейного объекта управления, описываемого системой из $n$ обыкновенных дифференциальных уравнений, является наличие в $\Omega$ матрицы управляемости $\boldsymbol{G}=\left|\boldsymbol{Y}, \boldsymbol{L}_{\boldsymbol{X}} \boldsymbol{Y}, \ldots, \boldsymbol{L}_{\boldsymbol{X}} \boldsymbol{Y}^{n-2}, \boldsymbol{L}_{\boldsymbol{X}} \boldsymbol{Y}^{n-1}\right|$ ранга $n$ (det $\boldsymbol{G}$ может обращаться в нуль в начале координат) и инволютивного множества $\left\{\boldsymbol{Y}, \boldsymbol{L}_{\boldsymbol{X}} \boldsymbol{Y}, \ldots, \boldsymbol{L}_{\boldsymbol{X}} \boldsymbol{Y}^{n-2}\right\}$, составленного из 
первых $(n-1)$ столбцов матрицы $\boldsymbol{G}$. Здесь $\boldsymbol{X}, \boldsymbol{Y}$ - векторные поля, связанные с линеаризируемой системой дифференциальных уравнений; $\boldsymbol{L}_{\boldsymbol{X}} \boldsymbol{Y}$ - производная Ли векторного поля $\boldsymbol{Y}$ вдоль векторного поля $\boldsymbol{X}$; $\boldsymbol{L}_{\boldsymbol{X}}^{n-2} \boldsymbol{Y}, \boldsymbol{L}_{\boldsymbol{X}}^{n-1} \boldsymbol{Y}$ - производные Ли $(n-2)$-го и $(n-1)$-го порядков поля $\boldsymbol{Y}$ вдоль векторного поля $\boldsymbol{X}[6,7]$.

С системой дифференциальных уравнений (2) связаны векторные поля:

$$
\begin{gathered}
\boldsymbol{X}(\boldsymbol{x})=\left|\begin{array}{c}
a_{10}+a_{11} x_{1}+a_{12} x_{2}+a_{13} x_{3}+a_{131} x_{3} e^{\beta x_{1}}=f_{1} \\
a_{21} x_{1}+a_{22} x_{2}=f_{2} \\
a_{30}+a_{31} x_{1}+a_{311} x_{1} e^{\beta x_{1}}+a_{33} x_{3}+a_{333} x_{3}^{2}=f_{3}
\end{array}\right| ; \\
\boldsymbol{Y}(\boldsymbol{x})=\left|\begin{array}{c}
a_{11 u} x_{1}=y_{1} \\
0=y_{2} \\
0=y_{3}
\end{array}\right|,
\end{gathered}
$$

где $\boldsymbol{x}=\left(x_{1}, x_{2}, x_{3}\right)$

Из условия линеаризации нелинейного объекта управления следует, что для линеаризации системы уравнений (2) обратной связью в пространстве "вход-состояние" необходимо, чтобы скобки Ли от двух первых столбцов матрицы управляемости $\boldsymbol{G}=\left|\boldsymbol{Y}, \boldsymbol{L}_{\boldsymbol{X}} \boldsymbol{Y}, \boldsymbol{L}_{\boldsymbol{X}}^{2} \boldsymbol{Y}\right|$ были равны нулю при ранге матрицы управляемости, равном трем [6, 7].

Вычислим первую производную Ли:

$$
\begin{aligned}
& \boldsymbol{L}_{\boldsymbol{X}} \boldsymbol{Y}=\frac{d \boldsymbol{Y}}{d \boldsymbol{x}} \boldsymbol{X}-\frac{d \boldsymbol{X}}{d \boldsymbol{x}} \boldsymbol{Y}=\left|\begin{array}{lll}
\frac{\partial y_{1}}{\partial x_{1}} & \frac{\partial y_{1}}{\partial x_{2}} & \frac{\partial y_{1}}{\partial x_{3}} \\
\frac{\partial y_{2}}{\partial x_{1}} & \frac{\partial y_{2}}{\partial x_{2}} & \frac{\partial y_{2}}{\partial x_{3}} \\
\frac{\partial y_{3}}{\partial x_{1}} & \frac{\partial y_{3}}{\partial x_{2}} & \frac{\partial y_{3}}{\partial x_{3}}
\end{array}\right| \cdot\left|\begin{array}{l}
f_{1} \\
f_{2} \\
f_{3}
\end{array}\right|-\left|\begin{array}{lll}
\frac{\partial f_{1}}{\partial x_{1}} & \frac{\partial f_{1}}{\partial x_{2}} & \frac{\partial f_{1}}{\partial x_{3}} \\
\frac{\partial f_{2}}{\partial x_{1}} & \frac{\partial f_{2}}{\partial x_{2}} & \frac{\partial f_{2}}{\partial x_{3}} \\
\frac{\partial f_{3}}{\partial x_{1}} & \frac{\partial f_{3}}{\partial x_{2}} & \frac{\partial f_{3}}{\partial x_{3}}
\end{array}\right| \cdot\left|\begin{array}{l}
y_{1} \\
y_{2} \\
y_{3}
\end{array}\right|= \\
& =\left|\begin{array}{ccc}
a_{11 u} & 0 & 0 \\
0 & 0 & 0 \\
0 & 0 & 0
\end{array}\right| \cdot\left|\begin{array}{l}
f_{1} \\
f_{2} \\
f_{3}
\end{array}\right|-\left|\begin{array}{ccc}
a_{11}+a_{131} \beta x_{3} e^{\beta x_{1}} & a_{12} & a_{13}+a_{131} \beta e^{\beta x_{1}} \\
a_{21} & a_{22} & 0 \\
a_{31}+a_{311} e^{\beta x_{1}}+a_{311} \beta x_{1} e^{\beta x_{1}} & 0 & a_{33}+2 a_{333} x_{3}
\end{array}\right| . \\
& \cdot\left|\begin{array}{c}
a_{11 u} x_{1} \\
0 \\
0
\end{array}\right|=\left|\begin{array}{c}
a_{11 u}\left(a_{10}+a_{12} x_{2}+a_{13} x_{3}+a_{131} x_{3} e^{\beta x_{1}}-a_{131} \beta x_{1} x_{3} e^{\beta x_{1}}\right)=\varphi_{1} \\
-a_{11 u} a_{21} x_{1}=\varphi_{2} \\
-a_{11 u} x_{1}\left(a_{31}+a_{311} e^{\beta x_{1}}+a_{311} \beta x_{1} e^{\beta x_{1}}\right)=\varphi_{3}
\end{array}\right| .
\end{aligned}
$$


Зная первую производную Ли, можно вычислить скобки Ли $\left[\boldsymbol{Y}, \boldsymbol{L}_{\boldsymbol{X}} \boldsymbol{Y}\right] \quad$ и $\quad$ ранг матрицы $\boldsymbol{D}=\left|\boldsymbol{Y}, \boldsymbol{L}_{\boldsymbol{X}} \boldsymbol{Y},\left[\boldsymbol{Y}, \boldsymbol{L}_{\boldsymbol{X}} \boldsymbol{Y}\right]\right|$. Непосредственная проверка показывает, что скобки Ли $\left[\boldsymbol{Y}, \boldsymbol{L}_{\boldsymbol{X}} \boldsymbol{Y}\right]$ не равны нулю, а ранг матрицы $\boldsymbol{D}$ равен трем. Отсюда следует, что условия линеаризации обратной связью системы управления (2) в пространстве "входсостояние" не выполняются. Поэтому в канал управления необходимо вводить дополнительную переменную $x_{4}=u$, а в систему уравнений (2) дополнительное дифференциальное уравнение:

$$
\begin{aligned}
\frac{d x_{1}}{d t} & =a_{10}+a_{11} x_{1}+a_{12} x_{2}+a_{13} x_{3}+a_{131} x_{3} e^{\beta x_{1}}+a_{11 u} x_{1} x_{4} \\
\frac{d x_{2}}{d t} & =a_{21} x_{1}+a_{22} x_{2} \\
\frac{d x_{3}}{d t} & =a_{30}+a_{31} x_{1}+a_{311} x_{1} e^{\beta x_{1}}+a_{33} x_{3}+a_{333} x_{3}^{2} \\
\frac{d x_{4}}{d t} & =v
\end{aligned}
$$

где $v$ - новое управление.

Непосредственное вычисления производных и скобок Ли, аналогичные вышеприведенным, показывают, что условия линеаризации системы нелинейных дифференциальных уравнений (5) не выполняются. Введение одной, двух или любого числа новых переменных (дифференциальных уравнений) в систему уравнений (5) не позволяет линеаризовать обратной связью систему уравнений (5) в пространстве "вход-состояние". Во многом это связано с тем, что в правые части уравнений (5) входят одночлены с $e^{\beta x_{1}}$, описывающие кривую намагничивания материала двигателя. Учитывая, что в процессе разгона электропоезда двигатели постоянного тока основное время работают в режиме насыщения магнитного потока, то кривую намагничивания можно приблизить двумя отрезками прямых: $\mathrm{AB}$ и $\mathrm{BC}$ (рис. 2), а при решении задачи оптимизации режима разгона электропоезда использовать только отрезок ВС. Сравнение переходных процессов при различных интенсивных режимах разгона электропоезда при использовании экспоненциального приближения кривой намагничивания и отрезка $\mathrm{BC}$, приближающего кривую намагничивания в режиме насыщения магнитного потока, показало их несущественное отличие. 


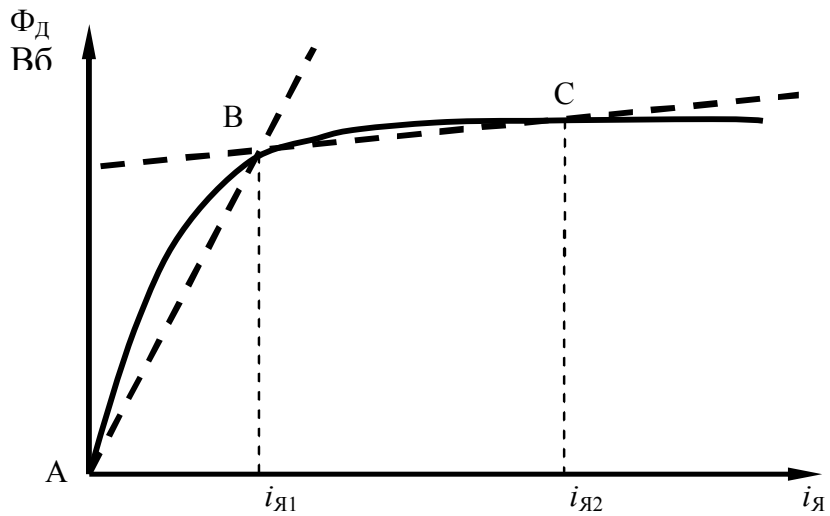

Рис. 2. Приближение кривой намагничивания электродвигателя постоянного тока

При упрощенном описании кривой намагничивания математическая модель (2) может быть записана в виде:

$$
\begin{aligned}
& \frac{d x_{1}}{d t}=b_{10}+b_{11} x_{1}+b_{12} x_{2}+b_{13} x_{3}+u_{1} ; \\
& \frac{d x_{2}}{d t}=b_{21} x_{1}+b_{22} x_{2} ; \\
& \frac{d x_{3}}{d t}=b_{30}+b_{31} x_{1}+b_{31} x_{1}+b_{33} x_{3}+b_{333} x_{3}^{2},
\end{aligned}
$$

где $b_{10}, b_{11}, \ldots, b_{333}$ - постоянные коэффициенты; $u_{1}=a_{11 u} x_{3} u$.

С системой дифференциальных уравнений (6) связаны векторные поля:

$$
\boldsymbol{X}_{1}=\left|\begin{array}{c}
b_{10}+b_{11} x_{1}+b_{12} x_{2}+b_{13} x_{3}=g_{1} \\
b_{21} x_{1}+b_{22} x_{2}=g_{2} \\
b_{30}+b_{31} x_{1}+b_{31} x_{1}+b_{33} x_{3}+b_{333} x_{3}^{2}=g_{3}
\end{array}\right|, \quad \boldsymbol{Y}_{1}=\left|\begin{array}{l}
1 \\
0 \\
0
\end{array}\right| \text {. }
$$

Для линеаризации системы уравнений (6) необходимо, чтобы скобки Ли от двух первых столбцов матрицы управляемости $\boldsymbol{G}=\left|\boldsymbol{Y}_{1}, \boldsymbol{L}_{\boldsymbol{X}_{1}} \boldsymbol{Y}_{1}, \boldsymbol{L}_{\boldsymbol{X}_{1}}^{2} \boldsymbol{Y}_{1}\right| \quad$ были равны нулю при ранге матрицы управляемости, равном трем [6, 7]. Имеем:

$$
\boldsymbol{L}_{\boldsymbol{X}_{1}} \boldsymbol{Y}_{1}=\frac{d \boldsymbol{Y}_{1}}{d \boldsymbol{x}} \boldsymbol{X}_{1}-\frac{d \boldsymbol{X}_{1}}{d \boldsymbol{x}} \boldsymbol{Y}_{1}=-\frac{d \boldsymbol{X}_{1}}{d \boldsymbol{x}} \boldsymbol{Y}_{1}=\left|\begin{array}{l}
-b_{11} \\
-b_{21} \\
-b_{31}
\end{array}\right|
$$




$$
\begin{array}{r}
\boldsymbol{L}_{\boldsymbol{X}_{1}}^{2} \boldsymbol{Y}_{1}=\boldsymbol{L}_{\boldsymbol{X}_{1}}\left(\boldsymbol{L}_{\boldsymbol{X}_{1}} \boldsymbol{Y}_{1}\right)=\frac{d\left(\boldsymbol{L}_{\boldsymbol{X}_{1}} \boldsymbol{Y}_{1}\right)}{d \boldsymbol{x}} \boldsymbol{X}_{1}-\frac{d \boldsymbol{X}_{1}}{d \boldsymbol{x}} \boldsymbol{L}_{\boldsymbol{X}_{1}} \boldsymbol{Y}_{1}=-\frac{d \boldsymbol{X}_{1}}{d \boldsymbol{x}} \boldsymbol{L}_{\boldsymbol{X}_{1}} \boldsymbol{Y}_{1}= \\
=-\left|\begin{array}{ccc}
b_{11} & b_{12} & b_{13} \\
b_{21} & b_{22} & 0 \\
b_{31} & 0 & b_{33}+2 b_{333} x_{3}
\end{array}\right| \cdot\left|\begin{array}{c}
-b_{11} \\
-b_{21} \\
-b_{31}
\end{array}\right|=\left|\begin{array}{c}
b_{11}^{2}+b_{12} b_{21}+b_{13} b_{31} \\
b_{21} b_{11}+b_{22} b_{21} \\
b_{31} b_{11}+b_{31}\left(b_{33}+2 b_{333} x_{3}\right)
\end{array}\right|,
\end{array}
$$

где $\boldsymbol{x}=\left(x_{1}, x_{2}, x_{3}\right)$.

Непосредственная проверка ранга матрицы управляемости показывает, что он равен трем, а скобки Ли $\left[\boldsymbol{Y}_{1}, \boldsymbol{L}_{\boldsymbol{X}_{1}} \boldsymbol{Y}_{1}\right]$ равны нулю, поскольку первые два столбца матрицы $\boldsymbol{G}$ являются постоянными. Таким образом, выполняются необходимые и достаточные условия для преобразования системы уравнений (6) к линейному виду в формуле Бруновского [6, 7]:

$$
\frac{d z_{1}}{d t}=z_{2} ; \frac{d z_{2}}{d t}=z_{3} ; \frac{d z_{3}}{d t}=v,
$$

где $z_{1}, z_{2}, z_{3}, v$ - переменные и управление в пространстве «вход состояние».

В этом случае существуют функции $T_{i}\left(x_{1}, x_{2}, x_{3}\right), i=\overline{1,3}, \mathrm{c}$ помощью которых осуществляется переход от переменных $x_{i}$ к переменным $z_{i}(i=\overline{1,3})$ :

$$
z_{i}=T_{i}\left(x_{1}, x_{2}, x_{3}\right), i=\overline{1,3} .
$$

Подставляя выражения (9) в систему уравнений (8), получим:

$$
\begin{gathered}
\frac{d z_{1}}{d t}=\frac{d T_{1}(\boldsymbol{x})}{d \boldsymbol{x}} \frac{d \boldsymbol{x}}{d t}=\frac{d T_{1}(\boldsymbol{x})}{d \boldsymbol{x}}\left(\boldsymbol{g}\left(g_{1}, g_{2}, g_{3}\right)+\boldsymbol{Y}_{1} u_{1}\right)=T_{2}(\boldsymbol{x}) ; \\
\frac{d z_{2}}{d t}=\frac{d T_{2}(\boldsymbol{x})}{d \boldsymbol{x}} \frac{d \boldsymbol{x}}{d t}=\frac{d T_{2}(\boldsymbol{x})}{d \boldsymbol{x}}\left(\boldsymbol{g}\left(g_{1}, g_{2}, g_{3}\right)+\boldsymbol{Y}_{1} u_{1}\right)=T_{3}(\boldsymbol{x}) ; \\
\frac{d z_{3}}{d t}=\frac{d T_{3}(\boldsymbol{x})}{d \boldsymbol{x}} \frac{d \boldsymbol{x}}{d t}=\frac{d T_{3}(\boldsymbol{x})}{d \boldsymbol{x}}\left(\boldsymbol{g}\left(g_{1}, g_{2}, g_{3}\right)+\boldsymbol{Y}_{1} u_{1}\right)=v .
\end{gathered}
$$

По теореме о линеаризации обратной связью по состоянию для функции $T_{1}$ должны выполнятся соотношения $[6,7]$ :

$$
\nabla T_{1}(\boldsymbol{x}) a d_{g}^{0} \boldsymbol{Y}_{1}=\nabla T_{1}(\boldsymbol{x}) \boldsymbol{Y}_{1}=0
$$




$$
\begin{aligned}
& \nabla T_{1}(\boldsymbol{x}) a d_{\mathbf{g}} \boldsymbol{Y}_{1}=0 ; \\
& \nabla T_{1}(\boldsymbol{x}) a d_{\mathbf{g}}^{2} \boldsymbol{Y}_{1} \neq 0,
\end{aligned}
$$

где $\nabla T_{1}(\boldsymbol{x})=\left(\frac{\partial T_{1}}{\partial x_{1}}, \frac{\partial T_{1}}{\partial x_{2}}, \frac{\partial T_{1}}{\partial x_{3}}\right) ; a d_{\boldsymbol{g}}^{0} \boldsymbol{Y}_{1}-$ скобки Ли нулевого порядка, равные самой функции $\boldsymbol{Y}_{1}$.

Из соотношений (13), (14) с учетом значений столбцов матрицы управляемости $\boldsymbol{G}$ имеем:

$$
\begin{aligned}
& \nabla T_{1}(\boldsymbol{x}) \boldsymbol{Y}_{1}=\left|\begin{array}{lll}
\frac{\partial T_{1}}{\partial x_{1}} & \frac{\partial T_{1}}{\partial x_{2}} & \frac{\partial T_{1}}{\partial x_{3}}
\end{array}\right| \cdot\left|\begin{array}{l}
1 \\
0 \\
0
\end{array}\right|=\frac{\partial T_{1}}{\partial x_{1}}=0 ; \\
& \nabla T_{1}(\boldsymbol{x}) a d_{\boldsymbol{g}} \boldsymbol{Y}_{1}=\left|\begin{array}{lll}
\frac{\partial T_{1}}{\partial x_{1}} & \frac{\partial T_{1}}{\partial x_{2}} & \frac{\partial T_{1}}{\partial x_{3}}
\end{array}\right| \cdot\left|\begin{array}{l}
-b_{11} \\
-b_{21} \\
-b_{31}
\end{array}\right|= \\
& =-b_{11} \frac{\partial T_{1}}{\partial x_{1}}-b_{21} \frac{\partial T_{1}}{\partial x_{2}}-b_{31} \frac{\partial T_{1}}{\partial x_{3}}=0 .
\end{aligned}
$$

Из выражения (16) следует, что функция $T_{1}$ не зависит от переменной $x_{1}$, поэтому уравнение в частных производных (17) можно записать в виде:

$$
b_{21} \frac{\partial T_{1}}{\partial x_{2}}+b_{31} \frac{\partial T_{1}}{\partial x_{3}}=0 .
$$

Одним из возможных решений уравнения (18) является следующее:

$$
T_{1}=x_{3}-\frac{b_{31}}{b_{21}} x_{2} .
$$

Проверим возможность использования этого решения. Вначале убедимся, что выполняется неравенство (15) при решении (19):

$$
\begin{aligned}
\nabla T_{1}(\boldsymbol{x}) a d_{\boldsymbol{g}}^{2} \boldsymbol{Y}_{1} & =\left|\begin{array}{lll}
0 & \frac{\partial T_{1}}{\partial x_{2}} & \frac{\partial T_{1}}{\partial x_{3}}
\end{array}\right| \cdot\left|\begin{array}{c}
b_{11}^{2}+b_{21} b_{12}+b_{13} b_{31} \\
b_{11} b_{21}+b_{21} b_{22} \\
b_{11} b_{31}+b_{31}\left(b_{33}+2 b_{333} x_{3}\right)
\end{array}\right|= \\
& =-b_{31} b_{22}+b_{31}\left(b_{33}+2 b_{333} x_{3}\right) \neq 0 .
\end{aligned}
$$

Таким образом, неравенство (15) выполняется. 
Зная $T_{1}$, несложно определить функции $T_{2}(\boldsymbol{x})$ и $T_{3}(\boldsymbol{x})$ :

$$
\begin{aligned}
& z_{2}=T_{2}(\boldsymbol{x})=L_{\boldsymbol{g}} T_{1}(\boldsymbol{x})=\nabla T_{1}(\boldsymbol{x}) \boldsymbol{g}(\boldsymbol{x})=\left|\begin{array}{lll}
0 & -\frac{b_{21}}{b_{31}} & 1
\end{array}\right| \cdot\left|\begin{array}{l}
g_{1} \\
g_{2} \\
g_{3}
\end{array}\right|= \\
& =b_{30}-\frac{b_{31} b_{22}}{b_{21}} x_{2}+b_{33} x_{3}+b_{333} x_{3}^{2} \\
& z_{3}=T_{3}(\boldsymbol{x})=L_{g} T_{2}(\boldsymbol{x})=\nabla T_{2}(\boldsymbol{x}) \boldsymbol{g}(\boldsymbol{x})=\left|\begin{array}{lll}
0 & \frac{\partial T_{2}}{\partial x_{2}} & \frac{\partial T_{2}}{\partial x_{3}}
\end{array}\right| \cdot\left|\begin{array}{l}
g_{1} \\
g_{2} \\
g_{3}
\end{array}\right|= \\
& =-\frac{b_{31} b_{22}}{b_{21}}\left(b_{21} x_{1}+b_{22} x_{2}\right)+\left(b_{33}+2 b_{333} x_{3}\right)\left(b_{30}+b_{31} x_{1}+b_{33} x_{3}+b_{333} x_{3}^{2}\right) \text {. }
\end{aligned}
$$

Для определения взаимосвязи между управлениями в исходной системе уравнений (6) и в системе уравнений в форме Бруновского (8) воспользуемся последним уравнение из системы (8)

$$
\frac{d z_{3}}{d t}=v
$$

или, так как $z_{3}=T_{3}$, то:

$$
\frac{d z_{3}}{d t}=\frac{d T_{3}}{d t}=\frac{d T_{3}}{d \boldsymbol{x}} \frac{d \boldsymbol{x}}{d t}=\frac{d T_{3}}{d \boldsymbol{x}}\left(\boldsymbol{g}+\boldsymbol{Y}_{1} u_{1}\right)=\frac{d T_{3}}{d \boldsymbol{x}} \boldsymbol{g}+\frac{d T_{3}}{d \boldsymbol{x}} \boldsymbol{Y}_{1} u_{1}=v .
$$

Поскольку

$$
\frac{d T_{3}}{d \boldsymbol{x}}=\boldsymbol{L}_{\boldsymbol{g}}^{3} T_{1} \text { и } \frac{d T_{3}}{d \boldsymbol{x}} \boldsymbol{Y}_{1}=\boldsymbol{L}_{\boldsymbol{Y}_{1}} \boldsymbol{L}_{\boldsymbol{g}}^{2} T_{1},
$$

то из (20) следует:

$$
u_{1}=\frac{v-\boldsymbol{L}_{g}^{3} T_{1}}{\boldsymbol{L}_{\boldsymbol{Y}_{1}} \boldsymbol{L}_{\boldsymbol{g}}^{2} T_{1}},
$$

то есть взаимосвязь между управлениями в разных моделях определена.

Синтез оптимальных управлений электропоездом с помощью системы управления в форме Бруновского и принципа максимума Понтрягина. Оптимизация процессов перевозки пассажиров электропоездами является одной из важных задач железнодорожного транспорта пригородного сообщения. В зависимости от специфических особенностей, предъявляемых к перевозке пассажиров, движению 
электропоездов, состояния пути и положения других составов могут быть сформулированы различные задачи оптимального управления электроприводом электропоезда. Несмотря на большое число таких задач, большинство из них может быть сведено к решению задач управления при минимизации или максимизации некоторого функционала. Наиболее часто в теории управления решается задача минимизации времени перевода объекта из одной точки фазового пространства в другую или, другими словами, задача максимального быстродействия. Эта задача важна и при управлении разгоном электропоезда, особенно в тех случаях, когда график движения составов весьма напряжен.

Решение задачи максимального быстродействия. Поскольку в форме Бруновского математическая модель объекта управления содержит всего три линейных дифференциальных уравнения, то задачу максимального быстродействия можно решить с помощью принципа максимума Понтрягина.

Сформулируем вначале задачу максимального быстродействия как задачу достижения максимальной скорости за минимальное время при управлении добавочным сопротивлением $R_{\text {д. }}$ В исходной системе уравнений (1), обороты эквивалентного электродвигателя, a, следовательно, и скорость движения электропоезда, определяется в результате интегрирования третьего дифференциального уравнения. В системе уравнений в форме Бруновского (8) переменной, прямо соответствующей скорости движения электропоезда нет. Однако переменная $z_{1}$ определяется соотношением (19):

$$
z_{1}=x_{3}-\frac{b_{31}}{b_{21}} x_{2},
$$

где $x_{3}$ - обороты электродвигателя, пропорциональные скорости $\boldsymbol{V}$ движения электропоезда:

$$
V=\frac{2 \pi x_{3}}{\mu} R_{\mathrm{K}}
$$

$\mu$ - передаточное отношение редуктора электродвигателя; $R_{\mathrm{K}}-$ радиус колеса колесной пары; $b_{31}, b_{21}$ - постоянные коэффициенты; $x_{2}$ - ток возбуждения.

В процессе разгона электропоезда ток возбуждения меняется не более чем на $20 \%$, поэтому в первом приближении можно полагать, что переменная $z_{1}$ в системе уравнений в форме Бруновского моделирует скорость движения состава. Поэтому при решении задачи максимального 
быстродействия необходимо достичь максимальной скорости $V_{\max }$ за минимальное время при управлении сопротивлением $R_{\text {д. }}$.

Из вида системы дифференциальных уравнений (8) в форме Бруновского и необходимости достижения максимального значения переменной $z_{1}$ следует, что управление $v$ в начальный момент движения должно принимать максимальное значение и оставаться таким до конца интервала управления, однако максимальное значение управления $\boldsymbol{v}$ (или

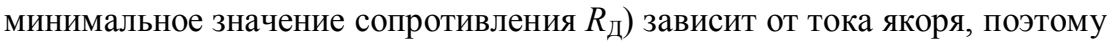
в каждый момент процесса разгона величина управления (величина сопротивления $R_{\text {Д}}$ ) определяется соотношением:

$$
v=v_{\max }\left(R_{\text {Я }}\right)
$$

или

$$
R_{\text {Д }}=R_{\text {Д } \min }\left(R_{\text {Я }}\right),
$$

где $v_{\max }\left(R_{Я}\right)$ - максимально возможное управление при заданном значении тока якоря; $R_{\text {Д min }}\left(R_{Я}\right)$ - минимально возможное значение сопротивления при заданном значении тока якоря.

Таким образом, решение задачи максимального быстродействия в этом случае тривиально и определяется в каждый момент времени предельно большим управлением $v$ (или предельно малым значением сопротивления $R_{\text {Д). }}$.

Решение задачи разгона электропоезда при ограничениях на расход управления и переходные динамические процессы. Система уравнений в форме Бруновского в этой задаче имеет вид:

$$
\frac{d z_{1}}{d t}=z_{2} ; \frac{d z_{2}}{d t}=z_{3} ; \frac{d z_{3}}{d t}=v .
$$

Требуется определить управление $v$, на которое наложено ограничение $v_{\min } \leq v \leq v_{\max }$, минимизирующее функционал

$$
J=\int_{t_{0}}^{t_{1}}\left(1+b_{0}\left(z_{30}-z_{3}(t)\right)^{2}+b_{1} v^{2}\right) d t,
$$

где $b_{0}, b_{1}, z_{30}$ - константы,

при разгоне электропоезда из начального состояния $z_{j}=0, j=\overline{1,3}$ в конечное состояние, в котором $z_{1}=z_{1 \max }\left(t_{1}\right)$, а на переменные $z_{2}$ и $z_{3}$ ограничения не заданы. 
Основные соотношения принципа максимума для объекта управления (8) и функционала (23) и имеют вид $[1,3,5]$ :

$$
\begin{gathered}
H\left(z_{j}(t), \Psi_{j}(t), v\right)=\Psi_{1} z_{2}+\Psi_{2} z_{3}+\Psi_{3} v+ \\
+\Psi_{0}\left[1+b_{0}\left(z_{30}-z_{3}(t)\right)^{2}+b_{1} v^{2}\right], j=\overline{0,3} ; \\
H\left(z_{j}(t), \Psi_{j}(t), v\right)=\max _{v \in G}\left(H\left(z_{j}(t), \Psi_{j}(t), v\right)\right), j=\overline{0,3} ; \\
\frac{d \mathrm{z}_{j}}{d t}=\frac{\partial H}{\partial \Psi_{j}} ; z_{j}\left(t_{0}\right)=0, j=\overline{0,3} ; \\
\frac{d \Psi_{j}}{d t}=-\frac{\partial H}{\partial z_{j}} ; \Psi_{j}\left(t_{1}\right)=\Psi_{j_{1}}, j=\overline{0,3} ;
\end{gathered}
$$

где $G$ - область допустимых управлений, задаваемая интервалом $\left[v_{\min }, v_{\max }\right] ; z_{j}\left(t_{0}\right), j=\overline{0,3}-$ значения фазовых координат в начальный момент времени; $\Psi_{j}\left(t_{1}\right), j=\overline{0,3}$ - значения сопряженных переменных в конечный момент интервала управления.

Если управление $v$ не принимает своих граничных значений $v_{\min }, v_{\max }$ и находится внутри интервала управления $\left[v_{\min }, v_{\max }\right]$, тогда справедливы выражения:

$$
\begin{gathered}
\Phi=\frac{\partial H}{\partial v}=\Psi_{3}+2 b_{1} v \Psi_{0}=0 ; \\
\Psi_{3}=-2 b_{1} v \Psi_{0} .
\end{gathered}
$$

Из теории принципа максимума [3, 5, 7] известно, что для нормальных вариационных задач, к которым относится и решаемая задача, сопряженные переменные определяются с точностью до произвольного постоянного множителя и обычно принимают, что $\Psi_{0}=-1$. Кроме того, для рассматриваемой задачи справедливо соотношение:

$$
H=\max _{v \in G} H\left(z_{j}, \Psi_{j}, v\right)=\text { const }=0 .
$$

Двух интервалов $Ф$ и $H$ уравнений движения не достаточно для исключения всех сопряженных переменных, однако с их помощью и с помощью скобок Пуассона $[H, \Phi]_{\mathrm{n}}$ можно получить третий интеграл движения [8]: 


$$
[H, \Phi]_{\mathrm{n}}=\sum_{j=1}^{3}\left(\frac{\partial H}{\partial z_{j}} \frac{\partial \Phi}{\partial \Psi_{j}}-\frac{\partial H}{\partial \Psi_{j}} \frac{\partial \Phi}{\partial z_{j}}\right)=0 .
$$

Так как функция Ф не зависит от $z_{j}(j=\overline{1,3})$ и зависит только от $\Psi_{3}$, то в сумме (29) только одно слагаемое из шести не равно нулю:

$$
[H, \Phi]_{\mathrm{n}}=\frac{\partial H}{\partial z_{3}} \frac{\partial \Phi}{\partial \Psi_{3}}=\left[\Psi_{2}+2 b_{0}\left(z_{30}-z_{3}(t)\right)\right] \cdot 1 .
$$

Поскольку из выражения (29) скобки Пуассона равны нулю, то имеем:

$$
\Psi_{2}=-2 b_{0}\left(z_{30}-z_{3}(t)\right) .
$$

Сопряженные переменные могут быть получены и другим способом - из уравнений (27):

$$
\begin{gathered}
\frac{d \Psi_{1}}{d t}=-\frac{\partial H}{\partial z_{1}}=0 \text { и } \Psi_{1}=\text { const }=C_{1} ; \\
\frac{d \Psi_{2}}{d t}=-\frac{\partial H}{\partial z_{2}}=-\Psi_{1} \text { и } \Psi_{2}=-C_{1} t+C_{2} ; \\
\frac{d \Psi_{3}}{d t}=-\frac{\partial H}{\partial z_{3}}=-\Psi_{2}+2 b_{0} \Psi_{0}\left(z_{30}-z_{3}(t)\right) .
\end{gathered}
$$

Из соотношений (30) и (32) следует, что

$$
-2 b_{0} z_{30}+2 b_{0} z_{3}(t)=C_{2}-C_{1} t
$$

Приравнивая свободные члены и члены, зависящие от времени, получим:

$$
\begin{gathered}
C_{2}=-2 b_{0} z_{30} ; \\
C_{1}=-2 b_{0} \frac{z_{1}(t)}{t} .
\end{gathered}
$$

Поскольку $C_{1}-$ константа, то это возможно только при $z_{1}(t)=k_{1} t$, поэтому имеем $C_{1}=-2 b_{0} k_{1}=\Psi_{1}$.

Зная $\Psi_{j}(j=\overline{0,3})$ из (24) и (28) можно определить управление $v$ :

$$
-2 b_{0} k_{1} z_{2}-2 b_{0}\left(z_{30}-z_{3}(t)\right) z_{3}+2 b_{1} v^{2}-\left[1+b_{0}\left(z_{30}-z_{3}(t)\right)^{2}+b_{1} v^{2}\right]=0
$$

или 


$$
v^{2}=\frac{1}{b_{1}}\left[2 b_{0} k_{1} z_{2}+2 b_{0}\left(z_{30}-z_{3}(t)\right) z_{3}+1+b_{0}\left(z_{30}-z_{3}(t)\right)^{2}\right] .
$$

Отсюда получаем:

$$
v=\sqrt{\frac{1}{b_{1}}+\frac{2 b_{0}}{b_{1}} k_{1} z_{2}+\frac{2 b_{0}}{b_{1}}\left(z_{30}-z_{3}(t)\right) z_{3}+\frac{b_{0}}{b_{1}}\left(z_{30}-z_{3}(t)\right)^{2}} .
$$

Поскольку значение подкоренного выражения в (34) может превышать $v_{\max }^{2}$, то соотношение (34) необходимо уточнить:

$$
v=\left\{\begin{array}{l}
\sqrt{D}, \text { если } \sqrt{D} \leq v_{\max } \\
v_{\max }, \text { если } \sqrt{D}>v_{\max },
\end{array}\right.
$$

где $D=\frac{1}{b_{1}}+\frac{2 b_{0}}{b_{1}} k_{1} z_{2}+\frac{2 b_{0}}{b_{1}}\left(z_{30}-z_{3}(t)\right) z_{3}+\frac{b_{0}}{b_{1}}\left(z_{30}-z_{3}(t)\right)^{2}$.

Если $\sqrt{D} \geq v_{\max }$ во всем интервале управления, то решение рассматриваемой задачи не отличается от задачи максимального быстродействия.

При $b_{0}=0$ получаем, что $D=\frac{1}{b_{1}}$, то есть, в этом случае, закон управления совпадает с законом управления при решении задачи максимального быстродействия при ограничениях на расход управления.

Выводы: таким образом, средствами геометрической теории управления впервые получена линейная модель электропривода электропоезда с двигателями постоянного тока, эквивалентная нелинейной модели привода. Показано, что с ее помощью можно решать задачи оптимального управления движением электропоезда.

Список литературы: 1. Моделирование и оптимизация систем управления и контроля локомотивов / В.И. Носков, В.Д. Дмитриенко, Н.И. Заполовский, С.Ю. Леонов. - Харьков: ХФИ "Транспорт Украины", 2003. - 248 с. 2. Красовский А.А., Буков В.И., Шендрик В.С. Универсальные алгоритмы оптимального управления непрерывными процессами. - М.: Наука, 1977. - 272 с. 3. Справочник по теории автоматического управления / Под редакцией А.А. Красовского. - М.: Наука. 1987. - 712 с. 4. Методы классической и современной теории автоматического управления: учебник в 5-ти томах. Т.5: Методы современной теории управления / Под ред. К.А. Пупкова, Н.Д. Егупова. - М.: МГТУ им. Н.Э. Баумана, 2005. 784 с. 5. Методы классической и современной теории автоматического управления: учебник в 5-ти томах. Т.4: Теория оптимизации систем автоматического управления / Под ред. К.А. Пупкова, Н.Д. Егупова. - М.: МГТУ им. Н.Э. Баумана, 2004. - 744 с. 6. Краснощеченко В.И., Грищенко А.П. Нелинейные системы: геометрический метод анализа и синтеза. - М.: МГТУ им. Н.Э. Баумана, 2005. - 520 с. 7. Ким Д.П. Теория автоматического управления: учебное пособие в 2-х томах. Т.2: Многомерные, нелинейные, 
оптимальные и адаптивные системы. - М.: Физматлит, 2004. - 464 с. 8. Ландау Л.Д., Лившиц Е.М. Теоретическая физика. В 10 томах. Т.1. Механика. - М.: Наука, 1988. 215 с.

\section{УДК 629.42}

Визначення оптимальних законів керування процесами руху електропоїзда / Дмитріснко В.Д., Носков В.І, Блиндюк В.С., Ліпчанський М.В., Нестеренко А.О. // Вісник НТУ "ХПІ". Серія: Інформатика та моделювання. - Харків: НТУ "ХПІ". - 2012. № 38. - С. $55-69$.

Вирішено задачу лінеаризації зворотнім зв'язком за станом нелінійної математичної моделі електропоїзда 3 тяговими двигунами постійного струму. На лінійній математичній моделі електропоїзда у формі Бруновського вирішені задачі оптимального керування при розгоні електропоїзда. Іл.: 2. Бібліогр.: 8.

Ключові слова: лінеаризація зворотним зв'язком за станом, математична модель електропоїзда, задачі оптимального керування.

\section{UDC 629.42}

Determination of the optimal control's laws of the electric train movement processes / Dmitrienko V.D., Noskov V.I., Blindyuk V.S., Lipchansky M.V., Nesterenko A.O. // Herald of the National Technical University "KhPI". Subject issue: Information Science and Modelling. Kharkov: NTU "KhPI". - 2012. - № 38. - P. 55 - 69.

The problem of the feedback linearization by the state of the electric trains's nonlinear mathematical model with DC traction motors is solved. The problems of the optimal control during the acceleration of the electric train are solved on the linear mathematical model in the Brunovsky form. Figs.: 2. Refs.: 8.

Keywords: feedback linearization by the state, the mathematical model of the electric train, optimal control problems.

Поступила в редакцию 09.06.2012 